\title{
Generation of Gene-Engineered Human Hepatoma Cells with Heat-Inducible Liver Functions
}

\author{
Hiroyuki KITANO ${ }^{1}$, Manuel SOUVERVIELLE SOTO ${ }^{2}$, Yuto SONODA ${ }^{1}$, \\ Yoshinori KAWABE $^{1}$, Akira ITO ${ }^{1}$, and Masamichi KAMIHIRA ${ }^{1,2^{*}}$ \\ ${ }^{1}$ Department of Chemical Engineering, Faculty of Engineering, Kyushu University, 744 Motooka, Nishi-ku, Fukuoka 819-0395, \\ Japan \\ ${ }^{2}$ Graduate School of Systems Life Sciences, Kyushu University, 744 Motooka, Nishi-ku, Nishi-ku, Fukuoka 819-0395, Japan
}

\begin{abstract}
Hepatoma cells derived from liver carcinoma are a candidate cell source for bioartificial liver (BAL) systems due to their high proliferative capacity, although liver function of hepatoma cells is considerably low compared with primary hepatocytes. In our previous study, genetically engineered mouse hepatoma cells with inducible high liver function were established by transducing liver-enriched transcription factor (LETF) genes. In this study, we aimed to develop new gene-engineered human hepatoma cells, in which high liver functions are inducible by heat treatment. For this purpose, we constructed a gene expression system for eight LETF genes under control of tetracycline-dependent transactivator (tTA), and the system was introduced into the genome of HepG2-HSP cells, in which a tTA expression system induced by a heat-shock protein promoter with transcriptional amplification was introduced into HepG2 cells. Thus, the heat-inducible tTA promotes LETF genes to induce liver function. Upon the heat treatment of the cells (HepG2-HSP/8F) at $43^{\circ} \mathrm{C}$ for $30 \mathrm{~min}$, liver functions such as albumin secretion and cytochrome P450 were significantly enhanced. The cells with heat-inducible liver function can be used as a new cell source for various hepatic studies including construction of BAL systems.
\end{abstract}

\section{Introduction}

The liver is the largest organ in the human body and has many functions that are essential to the maintenance of our lives. Although liver transplantation is the only effective treatment for acute and chronic liver failure (Neuberger, 2000), the shortage of donor organs is a serious problem. Cell transplantation and bioartificial liver (BAL) devices have been studied as treatments to complement and replace liver transplantation (Chamuleau et al., 2005). A BAL device is an extracorporeal support system in which functional hepatic cells are filled into a bioreactor. The hepatic cells metabolize xenobiotics and secret bioactive substances into the blood, and hence supporting liver function and promoting liver regeneration. Although several BAL systems have been proposed to use for clinical application (Lee et al., 2016), development for practical use has not progressed mainly due to the lack of suitable cells to be loaded.

Although human primary hepatocytes are an ideal cell source for the BAL systems, it is difficult to culture them for long period with maintaining high liver function in vitro. In recent years, it has been reported that some small molecules can convert rodent mature hepatocytes into liver progenitor cells (Katsuda et al.,
2017). Induction of three-dimensional miniaturized liver buds consisting of three progenitor (hepatic, vascular and mesenchymal cells) populations differentiated from human induced pluripotent stem (iPS) cells has also been reported (Takebe et al., 2017). However, culture and differentiation of stem/progenitor cells are timeconsuming and costly processes.

Hepatoma cells derived from liver carcinoma can be alternative cell sources for BAL systems. Hepatoma cells have high proliferation ability and can be cultured easily and inexpensively. However, the expression level of liver function in hepatoma cells is significantly low compared with primary hepatocytes. We have previously established a gene-engineered mouse hepatoma cell line that exhibits inducible liver function, by overexpression of eight liver-enriched transcription factors (LETFs), hepatocyte nuclear factor (HNF)-1 $\alpha$, HNF-1 $\beta$, HNF-3 $\beta$ (FOXA2), HNF-4 $\alpha$, HNF-6, CCAAT/enhancer binding protein (C/EBP)- $\alpha, \mathrm{C} / \mathrm{EBP}-\beta$ and $\mathrm{C} / \mathrm{EBP}-\gamma$ (Yamamoto et al., 2012; Yamamoto et al., 2018). In this study, human hepatoma HepG2 cells were genetically engineered to express high liver function by heat treatment. For this, genes encoding the human-derived eight LETFs were introduced into HepG2-HSP cells (Ito et al., 2019) as tTA-based transactivator-inducible expression cassettes.

*Corresponding author: kamihira@chem-eng.kyushu-u.ac.jp 


\section{Materials and Methods}

\subsection{Cell culture}

Hepatoma (HepG2, HepG2-HSP and HepG2-HSP/8F) cells were cultured in $10 \mathrm{~mL}$ high-glucose Dulbecco's modified medium (DMEM) (Sigma-Aldrich) supplemented with $10 \%$ fetal bovine serum (FBS), 0.1 $\mathrm{mg} / \mathrm{mL}$ streptomycin sulfate and $100 \mathrm{U} / \mathrm{mL}$ penicillin $\mathrm{G}$ potassium (Wako Pure Chemical Industries) in $100 \mathrm{~mm}$ collagen type I coated dishes (Iwaki). Cells were incubated at $37^{\circ} \mathrm{C}$ in a $5 \%(\mathrm{v} / \mathrm{v}) \mathrm{CO}_{2}$ incubator.

\subsection{Heat-shock condition examination}

HepG2-HSP cells were seeded into wells of 24-well collagen type I coated plates (Iwaki) at a density of $4.0 \times$ $10^{4}$ cells/well in medium. Next day (day 0 ), cells were heated at 41,42 or $43^{\circ} \mathrm{C}$ for 0.5 or $1.0 \mathrm{~h}$ using a water bath. On day 4, viable cell number and EGFP-positive cell rate were measured. Viable cell number was counted by the trypan blue dye staining. EGFP-positive cell analysis was performed using a cell sorter SH800 (Sony).

\subsection{Construction of transposon vector plasmids}

To introduce a tTA transactivator-mediated eight LETF genes expression system, two transposon vectors were constructed. The plasmids pUC57/pHSP-tTA-TRE-tTA and pUC57/TRE-hHNF1 $\alpha$ were also synthesized by Genewiz Inc. Five plasmids pMA-T/hCEBP $\alpha$-hCEBP $\beta-$ hCEBP $\gamma$-EF1 $\alpha$-Puro, pMA-T/hHNF3 $\beta$, pMK-RQBb/hHNF6-EF1 $\alpha$-Bla, pMK-RQ-Bb/hHNF4 $\alpha$ and pMA$\mathrm{T} / \mathrm{hHNF} 1 \beta$ were all synthesized by Invitrogen Inc. First, PB513B-1/pHSP-tTA-TRE-tTA was generated by ligation of SpeI- and EcoRV-digested pUC57/pHSPtTA-TRE-tTA into SpeI-digested PB513B-1 (SBI). To construct PB513B-1/TRE-hHNF1 $\alpha$-hHNF3 $\beta$-hCEBP $\alpha$ hCEBP $\beta$-hCEBP $\gamma$-EF1 $\alpha$-Puro, SpeI- and NheI-digested pMA-T/hCEBP $\alpha$-hCEBP $\beta$-hCEBP $\gamma$ was ligated into SpeI- and NheI-digested PB513B-1/pHSP-tTA-TRE-tTA to generate $\mathrm{PB} 513 \mathrm{~B}-1 / \mathrm{hCEBP} \alpha-\mathrm{hCEBP} \beta-\mathrm{hCEBP} \gamma-$ EF1 $\alpha$-Puro. The PB513B-1/hHNF3 $\beta$-hCEBP $\alpha$-hCEBP $\beta$ hCEBP $\gamma$-EF $1 \alpha$-Puro was then generated by ligation of $B g l$ II- and EcoRI-digested pMA-T/hHNF3 $\beta$. PB513B1/TRE-hHNF $1 \alpha$-hHNF3 $\beta$-hCEBP $\alpha$-hCEBP $\beta$-hCEBP $\gamma$ EF1 $\alpha$-Puro was constructed by insertion of SpeI- and BamHI-digested pUC57-Kan/TRE-hHNF1 $\alpha$ into SpeIand BamHI-digested PB513B-1/hHNF3 $\beta$-hCEBP $\alpha-$ hCEBP $\beta$-hCEBP $\gamma$-EF1 $\alpha$-Puro. For the construction of PB513B-1/TRE-hHNF1 $\beta$-hHNF4 $\alpha$-hHNF6-EF1 $\alpha$-Bla, $X b a \mathrm{I}-$ and HpaI-digested pMK-RQ-Bb/hHNF6-EF1 $\alpha$ Bla was ligated into $X b a \mathrm{I}-$ and $H p a \mathrm{I}$-digested PB513B1/pHSP-tTA-TRE-tTA to generate to generate PB513B1/pHSP-hHNF6-EF1 $\alpha$-Bla. Next, BglII- and EcoRIdigested pMK-RQ-Bb/hHNF4 $\alpha$ inserted into BglII- and
EcoRI-digested PB513B-1/pHSP-hHNF6-EF1 $\alpha$-Bla, resulting in PB513B-1/pHSP-hHNF4 $\alpha$-hHNF6-EF1 $\alpha$ Bla. Thereafter, PB513B-1/pHSP-hHNF1 $\beta$-hHNF4 $\alpha$ hHNF6-EF1 $\alpha$-Bla was generated by ligation of $X b a \mathrm{I}-$ and BamHI-digested pMA-T/hHNF $1 \beta$ into $X b a \mathrm{I}-$ and $B g l$ II-digested PB513B-1/pHSP-hHNF4 $\alpha$-hHNF6-EF1 $\alpha$ Bla. PB513B-1/TRE-hHNF1 $\beta$-hHNF4 $\alpha$-hHNF6-EF1 $\alpha$ Bla was constructed by insertion of the TRE sequence from pUC57-Kan/TRE-hHNF1 $\alpha$ into SpeI- and XbaIdigested PB513B-1/pHSP-hHNF1 $\beta$-hHNF4 $\alpha$-hHNF6EF1 $\alpha$-Bla.

\subsection{Transfection and drug screening}

HepG2-HSP cells were seeded into wells of 6-well collagen type I coated plates (Iwaki) at a density of $4.0 \times$ $10^{5}$ cells/well in medium. Next day, two transposon vectors and transposase vector were transfected using Lipofectamine 3000 reagent (Invitrogen) according to the manufacturer's instructions. The following amounts of reagent were used: $3.22 \mu \mathrm{g}$ PB513B-1/TRE-hHNF1 $\alpha$ hHNF3 $\beta$-hCEBP $\alpha$-hCEBP $\beta$-hCEBP $\gamma$-EF1 $\alpha$-Puro, 2.78 $\mu \mathrm{g} \quad$ PB513B-1/TRE-hHNF1 $\beta$-hHNF4 $\alpha$-hHNF6-EF1 $\alpha$ Bla, $1.5 \mu \mathrm{g}$ transposase vector, $250 \mu \mathrm{L}$ Opti-MEM (Gibco), $3.75 \mu \mathrm{L}$ Lipofectamine 3000 , and $15 \mu \mathrm{L}$ P3000 reagent. The cells were incubated at $37^{\circ} \mathrm{C}$ for $4 \mathrm{~h}$, and then the medium was changed to fresh medium. The cells were treated with $10 \mu \mathrm{g} / \mathrm{mL}$ puromycin (Gibco) and $5 \mu \mathrm{g} / \mathrm{mL}$ blasticidin (Gibco) from day 6 to day 12 after transfection to screen transgenic cells (HepG2-HSP-8F)

\subsection{Liver function analyses}

Hepatoma cells were cultured in $1 \mathrm{~mL} /$ well medium with or without $1.0 \mu \mathrm{g} / \mathrm{mL}$ Doxycycline hyclate (Dox, SigmaAldrich) at $4.0 \times 10^{4}$ cells/well in 24-well collagen type I coated plates (Iwaki). Next day (day 0), cells were heated at $43^{\circ} \mathrm{C}$ for 30 min using a water bath for heat treatment. The medium was changed every other day. On day 5 , the culture medium was harvested and stored at $80^{\circ} \mathrm{C}$ for albumin secretion assay. For cytochrome P450 assay, the medium was changed to fresh medium containing 3.0 $\mu \mathrm{M}$ Luciferin-IPA (Promega) and the cells were incubated for further $1 \mathrm{~h}$. Then, the culture medium was harvested and stored at $-80^{\circ} \mathrm{C}$. The cells were passaged and viable cell number was counted by the trypan blue dye staining method using a hemocytometer. The secreted albumin for $24 \mathrm{~h}$ was measured by ELISA using a commercially available kit (Mouse Albumin ELISA Quantitation set; Bethyl) according to the manufacturer's protocol. Cytochrom P450 activity (CYP3A4) was measured using a luminescence-based assay kit (P450-Glo, CYP3A4 assay with luciferin-IPA; Promega) according to the manufacture's protocol. These functions were normalized to the number of cells. 


\section{Results}

To generate human hepatoma cells with heat-inducible high liver functions, two transposon vectors were constructed (Figure 1A). These vectors encode LETF genes that are expressed under control of an inducible promoter containing tTA binding site (TRE). After two transposon vectors were introduced into HepG2-HSP cells, transgenic cells (HepG2-HSP/8F) were screened by puromycin and blasticidin selection. In HepG2-HSP cells, the activation of a heat shock protein promoter by heat treatment triggers bicistronic expression of EGFP and tTA genes. Then the tTA protein binds and activates $\mathrm{TRE} / \mathrm{pCMVmin}$ promoter, which in turn induces further expression of EGFP and tTA genes, providing a transcriptional positive feedback loop (Figure 1B). By introducing LETF genes under control of $\mathrm{TRE} / \mathrm{pCMVmin}$ promoter, tTA protein produced by heat treatment promotes LETF gene expression to induce high liver functions.

A

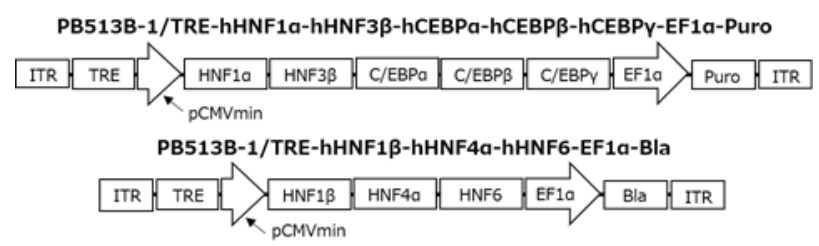

B

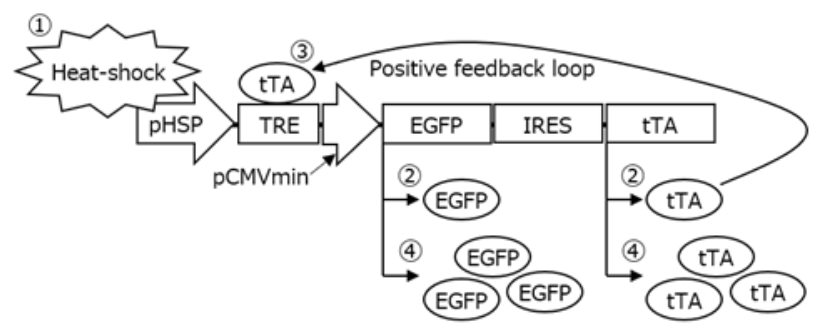

Figure 1. Heat-inducible gene expression system: (A) Construction of transposon vectors, (B) Gene expression system of HepG2-HSP cells

To determine heat treatment conditions to trigger the tTA positive feedback loop, HepG2-HSP cells were heated at 41,42 or $43^{\circ} \mathrm{C}$ for 0.5 or $1.0 \mathrm{~h}$. For each condition, proportion of EGFP-positive cells (Figure 2A) and viable cell number (Figure $2 \mathrm{~B}$ ) were measured. The percentage of EGFP-positive cells were $56.4 \%$ and $78.4 \%$ after heat treatment at $43^{\circ} \mathrm{C}$ for $1 \mathrm{~h}$ and $30 \mathrm{~min}$, respectively. Heat treatment at $43^{\circ} \mathrm{C}$ for $30 \mathrm{~min}$ did not affect cell proliferation, whereas cell proliferation decreased when cells were heated at $43^{\circ} \mathrm{C}$ for $1 \mathrm{~h}$. Therefore, the heat treatment for inducing overexpression of LETF genes was performed at $43^{\circ} \mathrm{C}$ for $30 \mathrm{~min}$.
A

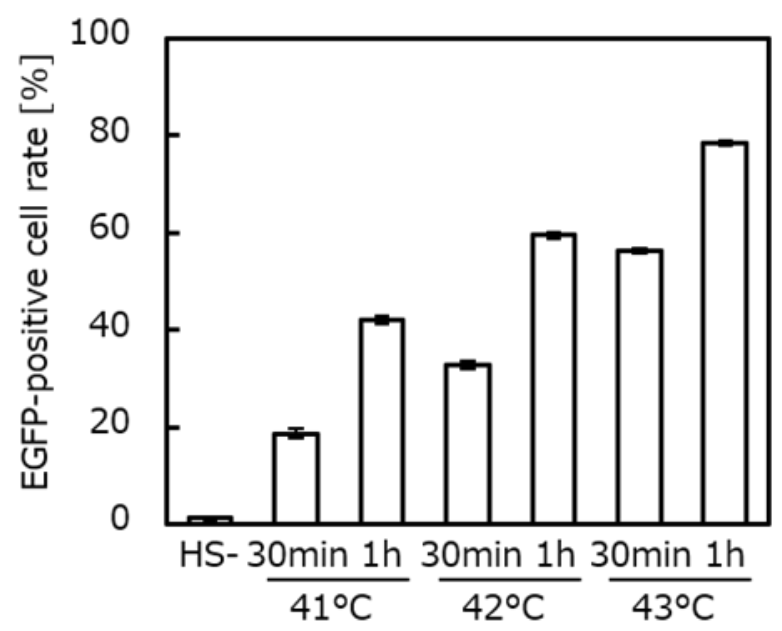

B

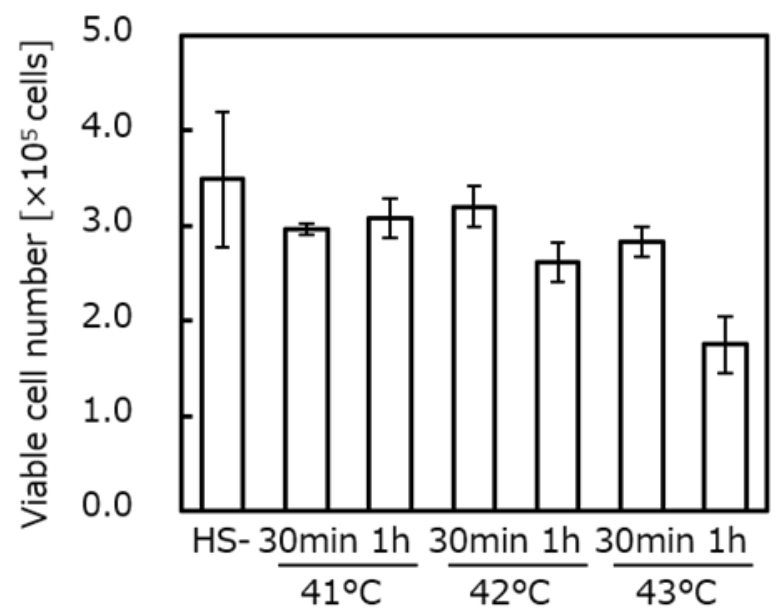

Figure 2. Heat-shock condition examination: (A) EGFPpositive cell rate, (B) Viable cell number

Next, the heat-shock response of HepG2-HSP/8F cells was evaluated. Cell number, albumin secretion rate, and cytochrome P450 activity were measured on day 5 of culture with or without heat treatment (Figure 3). The growth of parental HepG2-HSP cells remained unchanged with or without heat treatment, while the growth of HepG2-HSP/8F cells were significantly arrested upon overexpression of LETF by heat treatment (Figure 3A). Nevertheless, no obvious apoptotic cells were observed for $\mathrm{HepG} 2-\mathrm{HSP} / 8 \mathrm{~F}$ cells after heat treatment. As shown in Figure $3 \mathrm{~B}$ and $3 \mathrm{C}$, in HepG2$\mathrm{HSP} / 8 \mathrm{~F}$ cells with heat treatment, albumin secretion rate and CYP3A4 activity enhanced 2.6- and 5.3-fold compared with those of HepG2-HSP cells, respectively.

*Corresponding author: kamihira@chem-eng.kyushu-u.ac.jp 
A

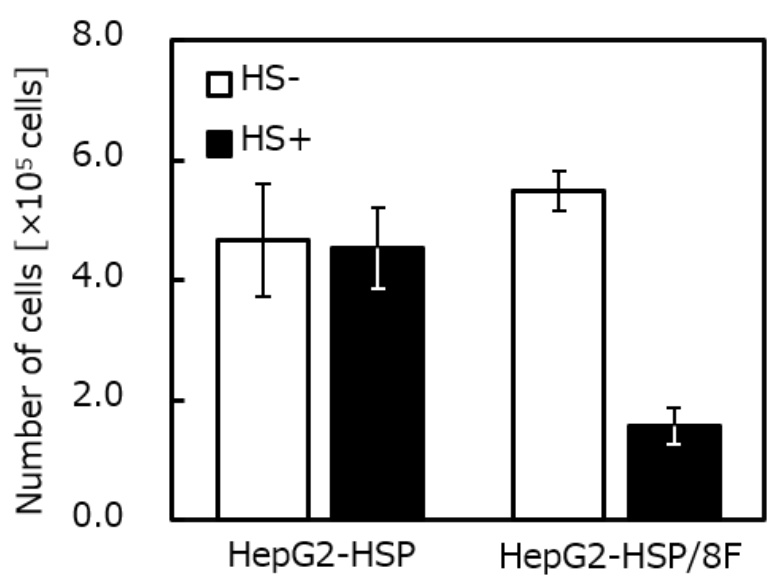

B

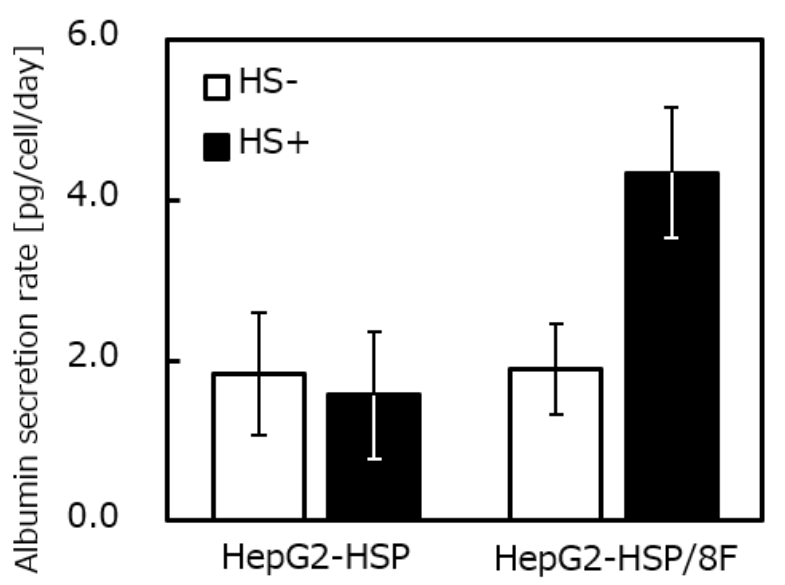

C

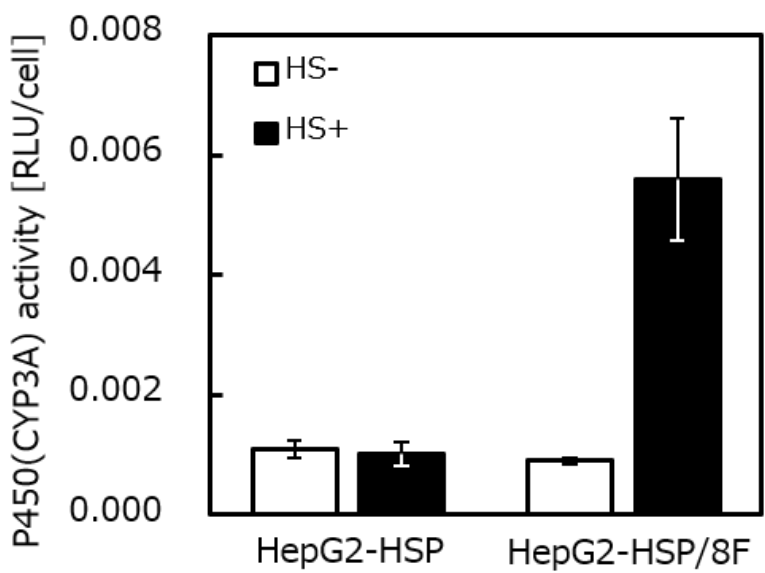

Figure 3. Liver function analyses: (A) Number of cells, (B) Albumin secretion rate, (C) CYP3A4 activity

\section{Discussion}

The clinical application of BAL systems has stagnated for a long time due to the unavailability of suitable cells. Human-derived hepatic cells with high liver function and proliferative capacity may be an ideal cell source.
However, it is difficult to control high liver function and proliferation in hepatic cells. Thus, the generation of cells capable of switching between proliferation and differentiation under normal culture conditions is of great importance for the development of BAL systems. In this study, we succeeded in creating a genetically engineered human hepatoma cell line, HepG2-HSP/8F, in which enhanced liver functions are induced by overexpression of eight LETF genes upon heat treatment. HepG2-HSP/8F cells readily proliferated in normal culture conditions. After induction by heat treatment, cells ceased proliferation and exhibited high albumin secretion and cytochrome P450 activity. Transposon vectors are heterogeneously introduced into cells and randomly insert genes into the cell genome. Thus, transduced cells without cloning are a mixture of cells with various expression levels of transgenes, and to exhibit the average level of expression. The genetically engineered mouse hepatoma Hepa/8F5 cells with inducible overexpression of eight LETF genes exhibited 13-fold higher albumin secretion compared with that of transgenic cell pool. Therefore, it may be possible to establish cell lines expressing high liver function by cloning of HepG2-HSP/8F cells.

In the liver, LETFs are highly expressed and a complex regulatory mechanism is involved in the expression of liver functions (Nagaki et al., 2008). LETFs regulate not only their various target genes related to liver functions but also themselves and mutual genes, forming a transcriptional network to express and maintain the hepatic phenotype (Hayashi et al., 1999). In our previous study, cells in which all eight LETFs were introduced the highest expression of liver function. Thus, induction of synergistic effects by overexpression of all transduced LETF genes is essential for differentiating hepatoma cells into mature hepatocytes. In this study, transposon vectors encoding drug resistance genes were used for screening cells transduced eight LETF genes, so that all LETFs are surely integrated into the genome of HepG2-HSP cells. Therefore, clonal cells isolated from HepG2-HSP/8F cells can be expected to express other liver functions and mature hepatocyte markers.

The functionality of hepatoma cells was significantly enhanced in three-dimensional culture such as spheroids, and the high liver function was maintained for long period (Landry et al., 1985; Hamamoto et al., 1998; Chang et al., 2009). In fact, Hepa/8F5 cells we established were able to improve liver functions by three-dimensional culture using macroporous gelatin beads (Tonello et al., 2107). However, as the culture period was extended and the beads became overconfluent with the cells, the level of liver function per cell gradually decreased due to the proliferation of cells. In this study, HepG2-HSP/8F cells were functionalized by heat treatment, allowing to induce liver function even after three-dimensional culture with high density and after filling into bioreactor devices.

In conclusion, we generated human hepatoma cells with inducible liver functions by heat treatment. The genetically engineered hepatoma cells would be a new resource for hepatic cell studies and for constructing BAL systems. 


\section{References}

Neuberger, J.; "Liver Transplantation," J. Hepatol., 32, 198-207 (2000)

Chamuleau, R. A., T. Deurholt, and R. Hoekstra; "Which Are the Right Cells to Be Used in a Bioartificial Liver," Metab. Brain Dis., 20, 327-335 (2005)

Lee, K. C., V. Stadlbauer, and R. Jalan; "Extracorporeal Liver Support Devices for Listed Patients," Liver Transpl., 22, 839-848 (2016)

Katsuda, T., M. Kawamata, K. Hagiwara, R. U. Takahashi, Y. Yamamoto, F. D. Camargo, and T. Ochiya; "Conversion of Terminally Committed Hepatocytes to Culturable Bipotent Progenitor Cells with Regenerative Capacity," Cell Stem Cell, 20, 41-55 (2017)

Takebe, T., K. Sekine, M. Kimura, E. Yoshizawa, S. Ayano, M. Koido, S. Funayama, N. Nakanishi, T. Hisai, T. Kobayashi, T. Kasai, R. Kitada, A. Mori, H. Ayabe, Y. Ejiri, N. Amimoto, Y. Yamazaki, S. Ogawa, M. Ishikawa, Y. Kiyota, Y. Sato, K. Nozawa, S. Okamoto, Y. Ueno, and H. Taniguchi; "Massive and Reproducible Production of Liver Buds Entirely from Human Pluripotent Stem Cells," Cell Rep., 21, 2661-2670 (2017)

Yamamoto, H., Y. Kawabe, A. Ito, and M. Kamihira; "Enhanced Liver Functions in Mouse Hepatoma Cells by Induced Overexpression of Liver-Enriched Transcription Factors," Biochem. Eng. J., 60, 67-73 (2012)

Yamamoto, H., J. M. Tonello, T. Sambuichi, Y. Kawabe, A. Ito, and M. Kamihira; "Characterization of Genetically Engineered Mouse Hepatoma Cells with Inducible Liver Functions by Overexpression of LiverEnriched Transcription Factors," J. Biosci. Bioeng., 125, 131-139 (2018)
Ito, A., R. Teranishi, K. Kamei, M. Yamaguchi, A. Ono, S. Masumoto, Y. Sonoda, M. Horie, Y. Kawabe, and M. Kamihira; "Magnetically Triggered Transgene Expression in Mammalian Cells by Localized Cellular Heating of Magnetic Nanoparticles," J. Biosci. Bioeng., 128, 355-364 (2019)

Nagaki, M. and H. Moriwaki; "Transcriptional Factor HNF and Hepatocyte Differentiation," Hepatol. Res., 38, 961-969 (2008)

Hayashi, Y., W. Wang, T. Ninomiya, H. Nagano, K. Ohta, and H. Itoh; "Liver Enriched Transcription Factors and Differentiation of Hepatocellular Carcinoma," Mol. Pathol., 52, 19-24 (1999)

Landry, J., D. Bernier, C. Ouellet, R. Goyette, and N. Marceau; "Spheroidal Aggregate Culture of Rat Liver Cells: Histotypic Reorganization, Biomatrix Deposition, and Maintenance of Functional Activities," J. Cell Biol., 101, 914-923 (1985)

Hamamoto, R., K. Yamada, M. Kamihira, and S. Ijima; "Differentiation and Proliferation of Primary Rat Hepatocytes Cultured as Spheroids," J. Biochem., 124, 972-929 (1998)

Chang, T. T. and M. Hughes-Fulford; "Monolayer and Spheroid Culture of Human Liver Hepatocellular Carcinoma Cell Line Cells Demonstrate Distinct Global Gene Expression Patterns and Functional Phenotypes," Tissue Eng. Part A, 15, 559-567 (2009)

Tonello, J. M., S. Kawashima, K. Sato, Y. Kawabe, A. Ito, and M. Kamihira; "Three-Dimensional Culture of a Genetically Modified Hepatoma Cell Line Using Macroporous Gelatin Beads," Cytotechnology, 69, 925931 (2017) 